\title{
AN AGGRESSIVE PRESENTATION OF PANTON VALENTINE LEUKOCIDIN POSITIVE STAPHYLOCOCCUS AUREUS AS AN OSTEOARTICULAR INFECTION IN A PREVIOUSLY HEALTHY 5-YEAR OLD
}

J. Ndzo1, K. Kamalanathan1.

1Southampton Children`s Hospital, Paediatrics, Southampton, United Kingdom

Introduction

Panton Valentine Leukocidin (PVL) Positive Staphylococcus aureus (SA) is an aggressive strain of SA that produces a toxin called PVL which causes cellular necrosis. This entity has not been well described in the UK, and is not well known by clinicians even though it is responsible for most cases of necrotic skin and soft tissue infections. The aim of this study was to describe the case of a 5-year old girl who presented to our hospital with a PVL positive SA infection, her management and her outcome.

\section{Case Presentation}

A previously healthy 5-year old girl presented to a district hospital with a 3-day history of fever and a swollen and painful right knee. She was born to Fijian parents; her dad was a soldier and mom a healthcare worker.

She was unwell and promptly started on Flucloxacillin after a diagnosis of septic arthritis of the right knee was made. Her inflammatory markers were raised, except her Neutrophil counts. The knee was also drained surgically with frank pus removed. She showed some clinical improvement but later deteriorated again. A repeat MRI did not show any collections, and a decision was made for a second wash-out was done and Clindamycin was added despite no evidence of more pus in the wound. Blood and fluid cultures revealed $\mathrm{PVL}$ positive $\mathrm{SA}$ at this point, and because she was not improving, a referral was made to our hospital, a tertiary facility. Upon arrival, she was reviewed by the ID team who added Linezolid to her antibiotic regimen with good effect.
5 days later her wound was noticed to discharge pus and she was again taken to theatre. She needed two blood transfusions and had a total of five more knee wash-outs, at which point the wound failed to close primarily, requiring a transfer to a center for plastic surgery where she had soft tissue coverage of the defect. She got discharged on Ceftriaxone, Clindamycin and Rifampicin to complete an 11-week course of antibiotics in total, 4 of which were intravenous.

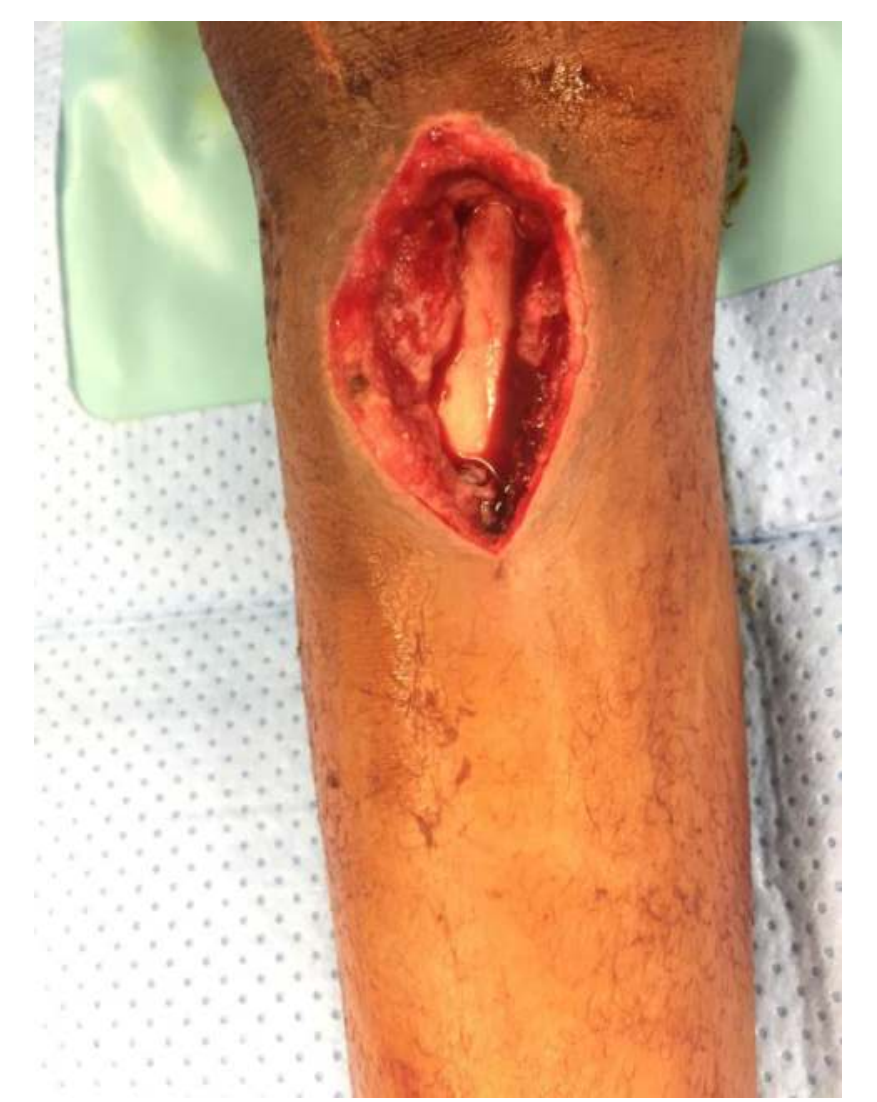

Three months after the initial infection, she came for a routine follow-up. Her wounds had healed and she was well in herself. Follow-up $x$-ray showed a moth-eaten appearance of her whole tibia. She is currently undergoing physiotherapy but will need long term followup to monitor for chronic osteomyelitis. Background
Discussion

This case is a classic demonstration of the aggressive nature of PVL positive SA Despite the early presentation and aggressive initial management, the patient still had a poor outcome.

The severity of the illness is compounded by the fact that the toxins trigger a release of free radicals in the tissues and neutrophils, impeding an immune response.

In a case series in the USA, patients with PVL positive SA had longer duration of hospitalisation, more deep-seated infections requiring multiple surgeries and higher morbidity and mortality compared to those with PVL negative SA.

The bacteria also accounts for almost al cases of necrotic pneumonia and a quarter of cases of acute osteomyelitis and as such, a high index of suspicion is necessary to diagnose infections.

Clinical signs to watch out for include unwell, septic patients, poor response to treatment despite appropriate therapy, and lack of neutrophilia despite an inflammatory response.

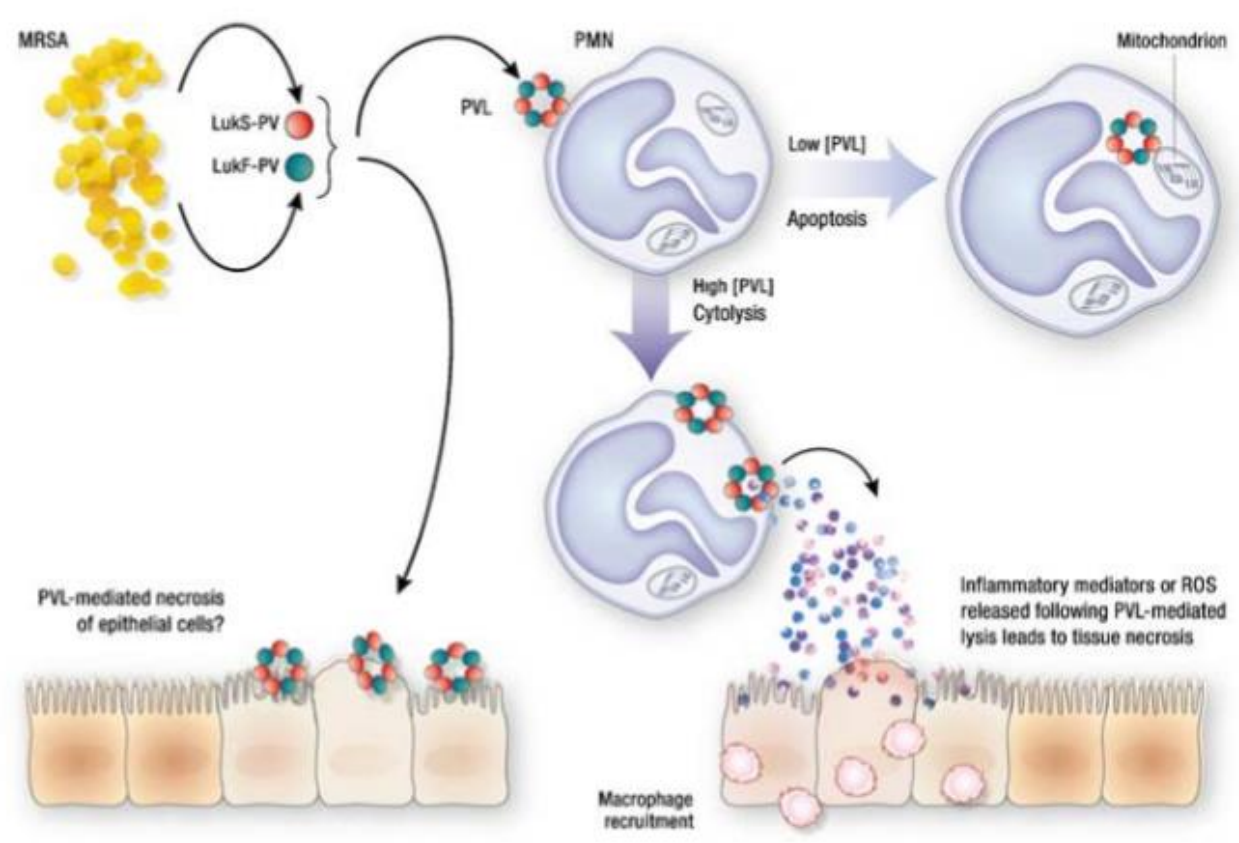

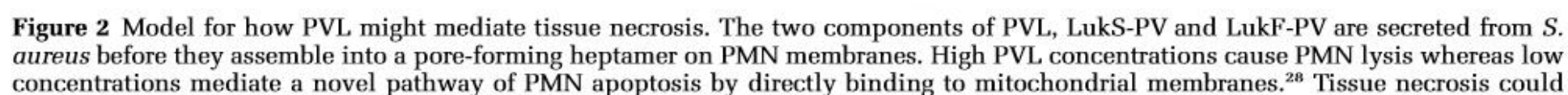
concentrations mediate a novel pathway of PMN apoptosis by directly binding to mitochondrial membraneses.2. Tissue necrosis could
result from release of reactive oxygen species (ROSS from lysed PMNS. Alternately release of granule contents from ysed PMN could set
in motion an inflammatory response, eventually resulting in tissue necrosis. It is unlikely that PVL has a direct necrotic effect on
1. Osteoarticular infections with PVL positive Staphylococcus aureus require aggressive medical and surgical treatment and could lead to potential long-term adverse effects. . Prompt diagnosis is vital as an antibiotics need to be modified. The involvement of the infectius disease team is mandatory, when available. 